\title{
Sportello del/al/per il cittadino
}

Ilaria Bonomi

PUBBLICATO: 27 MARZO 2020

\section{Quesito:}

Un interessante quesito ci viene posto dal sindaco del comune di Sernaglia della Battaglia, in provincia di Treviso, teatro di tragici eventi durante la prima guerra mondiale, e insignito dalla Presidenza della Repubblica nel 2009 di una Medaglia d'oro al merito civile per l'esemplare comportamento della popolazione. Il Comune ha istituito uno sportello al cittadino, della cui denominazione si chiede la correttezza linguistica.

\section{Sportello del/al/per il cittadino}

L 'uso delle tre diverse preposizioni, al/del/per il, in unione con il sostantivo sportello presenta leggere ma significative differenze di significato. Sportello al cittadino, espressione ellittica che sottintende rivolto, lascia intendere che questo servizio è concepito proprio per aiutare il cittadino nel suo rapporto con l'istituzione comunale e forse anche al di là di questo. Sportello per il cittadino non è molto distante dal precedente, ma sembra circoscrivere la funzione del servizio ai compiti istituzionali. Sportello del cittadino, infine, avvicina il destinatario del servizio all'istituzione in un rapporto piu diretto, presentando il cittadino non solo come destinatario ma quasi come possibile parte attiva. Tale specificità semantica è propria anche di espressioni come giornata (lunedi, martedi, ecc.) del cittadino, che vogliono sottolineare come in quella giornata, che offre apertura oraria dei servizi più ampia del solito, il cittadino sia facilitato nelle sue necessità.

Una breve ricerca con Google libri (fatta in data 9 dicembre 20r9) documenta come "sportello del cittadino" sia la più frequente con I.Igo occorrenze, particolarmente in ambito fiscale («Lo "sportello del cittadino" è un ufficio specializzato per accogliere il cittadino, per aiutarlo a capire cosa deve fare e dove può sbrigare le sue pratiche con il fisco») e in ambito forense ("Struttura volta a fornire informazioni e orientamento ai cittadini per la fruizione delle prestazioni professionali degli avvocati e per l'accesso alla giustizia"), ma anche in ambiti diversi.

"Sportello al cittadino" presenta 546 ricorrenze, tra le quali molte fanno riferimento all'interazione informatica, ma troviamo anche definizioni più generali come "figure di Sportello al cittadino, cioè una struttura organizzativa destinata a fornire servizi di front-office", con il ricorso all'anglicismo che indica in ambito aziendale 'l'insieme delle strutture di un'organizzazione che gestiscono l'interazione con il cliente'.

La terza espressione, "sportello per il cittadino", ricorre in misura ancora minore, 316 volte, per contesti e usi diversi, e anche, significativamente, in ambito forense, a indicare l'intercambiabilità con "sportello del cittadino".

Delle tre espressioni, dunque, appare decisamente più diffusa la prima, ma questo potrebbe anche modificarsi nel giro di qualche tempo. Le sfumature semantiche che abbiamo citato più sopra si possono anche non cogliere, considerando le tre espressioni del tutto equivalenti sul piano funzionale, e la documentazione statistica sembrerebbe confermarlo. Nessuna di esse, va sottolineato, è più o meno corretta delle altre dal punto di vista grammaticale: la lingua, in questo caso, consente una 
alternanza tra le preposizioni che in altri casi non consente, perché l'uso di ciascuna di esse risponde a vincoli grammaticali o a valori semantici specifici.

\section{Cita come:}

Ilaria Bonomi, Sportello del/al/per il cittadino , "Italiano digitale", 2019, XII, 2020/1 (gennaio-marzo) DOI: 10.35948/2532-9006/2020.3275

\section{Copyright 2019 Accademia della Crusca}

Pubblicato con licenza creative commons CC BY-NC-ND 